\title{
Modified Fractional Reduced Differential Transform Method for the Solution of Multiterm Time-Fractional Diffusion Equations
}

\author{
Salah Abuasad $\mathbb{D}^{1},{ }^{1}$ Ishak Hashim $\mathbb{D}^{2},{ }^{2}$ and Samsul Ariffin Abdul Karim $\mathbb{D}^{3}$ \\ ${ }^{1}$ Preparatory Year Deanship, King Faisal University, 31982 Hofuf, Al-Hasa, Saudi Arabia \\ ${ }^{2}$ School of Mathematical Sciences, Faculty of Science \& Technology, Universiti Kebangsaan Malaysia, UKM Bangi, \\ 43600 Selangor, Malaysia \\ ${ }^{3}$ Fundamental and Applied Sciences Department and Center for Smart Grid Energy Research (CSMER), \\ Institute of Autonomous System, Universiti Teknologi PETRONAS, Bandar Seri Iskandar, 32610 Seri Iskandar, Perak DR, Malaysia \\ Correspondence should be addressed to Salah Abuasad; sabuasad@kfu.edu.sa
}

Received 25 December 2018; Revised 31 March 2019; Accepted 8 April 2019; Published 2 May 2019

Academic Editor: Zine El Abiddine Fellah

Copyright (C) 2019 Salah Abuasad et al. This is an open access article distributed under the Creative Commons Attribution License, which permits unrestricted use, distribution, and reproduction in any medium, provided the original work is properly cited.

\begin{abstract}
In this study, we introduce a new modification of fractional reduced differential transform method (m-FRDTM) to find exact and approximate solutions for nonhomogeneous linear multiterm time-fractional diffusion equations (MT-TFDEs) of constant coefficients in a bounded domain with suitable initial conditions. Different applications in two and three fractional order terms are given to illustrate our new modification. The approximate solutions are given in the form of series solutions. The results show that the m-FRDTM for MT-TFDEs is a powerful method and can be generalized to other types of multiterm time-fractional equations.
\end{abstract}

\section{Introduction}

Researchers are trying to find different techniques to solve linear and nonlinear fractional differential equations. Some of them modify the classical methods to be an effective approach, others related between two or more methods to find numerical or analytical solutions of fractional equations. For instance, in $[1,2]$ we modified definition of beta fractional derivative to find exact and approximate solutions of time-fractional diffusion equations in different dimensions. Many numerical and analytical methods have been developed for single-term-fractional derivatives (only one fractional power). On the other hand, there are still little works in multi-term-fractional derivatives (more than one fractional power). The time-fractional diffusion-wave equation describes important physical phenomena that arise in amorphous, colloid, glassy, and porous materials, in fractals and percolation clusters, comb structures, dielectrics and semiconductors, biological systems, polymers, random and disordered media, and geophysical and geological processes [3]; Abuasad et al. [4] applied a fractional multistep differential transformed method to find approximate solutions to one of the most important epidemiologies and mathematical ecologies, fractional stochastic SIS epidemic model with imperfect vaccination. Recently, the extension to multiterm time-fractional diffusion equations (MT-TFDEs) has been considered [5]. Edwards et al. [6] showed that the numerical approximation of the solution of a linear multiterm fractional differential equation can be calculated by reduction of the problem to a system of ordinary and fractional differential equations each of order at most unity. Gejji and Bhalekar [7] solved multiterm linear and nonlinear diffusion-wave equations of fractional order using the Adomian decomposition method (ADM). In [8] they used the method of separation of variables to solve multiterm fractional diffusionwave equation subject to homogeneous/nonhomogeneous boundary conditions. Srivastava and Rai [9] used a new iterative method (NIM) and a modified Adomian decomposition method (MDM) to solve the MT-TFDE for different conditions. El-Sayed et al. [10] compared the Adomian decomposition method (ADM) against the proposed numerical method (PNM) to solve multiterm nonlinear fractional differential equations. Jiang et al. [3] used the method of separating variables to solve the multiterm timefractional diffusion-wave equation and the MT-TFDE. Jin et al. [11] analyzed a space semidiscrete scheme based on the 
standard Galerkin finite element method using continuous piecewise linear functions to solve an initial/boundary value problem for a diffusion equation, involving multiple timefractional derivatives. Dehghan et al. [12] applied a high order difference scheme and Galerkin spectral technique for the numerical solution of multiterm time-fractional partial differential equations. Zheng et al. [13] developed a high order numerical method based on the space-time spectral method for solving MT-TFDEs. Zhao et al. [5] used the finite element method to establish a fully discrete scheme for a class of two-dimensional MT-TFDEs. Chen et al. [14] proposed a unified numerical scheme based on the finite difference method in time and Legendre spectral method in space to solve MT-TFDE and diffusion-wave equations with variable coefficients; in this scheme they employed the Legendre spectral method in space and the weighted and shifted Grünwald difference operators for the discretization of the time-fractional operators.

Fractional reduced differential transform method (FRDTM) has been used successfully for solving linear and nonlinear fractional differential equations to obtain exact and approximate solutions. FRDTM was firstly introduced by Keskin and Oturance [15]. Gupta [16] considered eight different cases to find the approximate analytical solutions of Benney-Lin equation with fractional time derivative by FRDTM and the homotopy perturbation method (HPM). Srivastava et al. [17] studied the generalized time fractionalorder biological population model (GTFBPM) by FRDTM and Rawashdeh [18] employed FRDTM to solve the nonlinear fractional Harry-Dym equation. Singh and Srivastava [19] presented an approximate series solution of the multidimensional (heat-like) diffusion equation with time-fractional derivative using FRDTM. Saravanan and Magesh [20] compared two analytical methods: FRDTM vs fractional variational iteration method (FVIM), to find numerical solutions of the linear and nonlinear Fokker-Planck partial differential equations with space and time fractional derivatives, Singh [21] presented FRDTM to compute an alternative approximate solution of initial valued autonomous system of linear and nonlinear fractional partial differential equations. Rawashdeh [22] proposed FRDTM to solve one dimensional space and time fractional Burgers' equations (BE) and the time-fractional Cahn-Allen equation and Arshad et al. [23] presented a general form of FRDTM to solve wave-like problems, Zakharove-Kuznetsov equation, and couple BE, and Abuasad et al. [24] proposed FRDTM for finding exact and approximate solutions of the fractional Helmholtz equation.

In our work, we modify the FRDTM for the first time to handle MT-TFDEs. The rest of the paper is organized as follows: Section 2 gives some basic definitions and Section 3 presents the FRDTM for single and multiterm time-fractional equations. Three illustrative examples are given in Section 4. Section 5 is the conclusion.

\section{Preliminaries}

In this section, we give some basic definitions that we will use in the next sections.
2.1. The Gamma Function. The Gamma function $\Gamma(x)$ is simply a generalization of the factorial real arguments. The Gamma function can be defined as [25]

$$
\Gamma(z)=\int_{0}^{\infty} e^{-t} t^{z-1} \mathrm{~d} t, \quad z>0 .
$$

2.2. Fractional Derivative. There are various definitions for fractional derivatives. The three most frequently used definitions for the general fractional derivative are the GrünwaldLetnikov (GL) definition, the Riemann-Liouville (RL), and the Caputo definition ([25-27]). In this paper we will use the Caputo fractional derivative, since the initial conditions for fractional order differential equation are in a form involving only the limit values of integer-order derivative at the lower terminal initial time $(t=a)$, such as $y^{\prime}(a), y^{\prime \prime}(a), \ldots$ [25], and also the fractional derivative of a constant function is zero. The Caputo fractional derivative is defined as ([28])

$$
\begin{aligned}
& { }^{c} \mathbf{D}_{t}^{\alpha} f(t) \\
& := \begin{cases}\frac{1}{\Gamma(n-\alpha)} \int_{a}^{t} \frac{f^{(n)}(\tau)}{(t-\tau)^{\alpha+1-n}} \mathrm{~d} \tau, & n-1<\alpha<n, \\
\frac{\mathrm{d}^{n}}{\mathrm{~d} t^{n}} f(t), & \alpha=n,\end{cases}
\end{aligned}
$$

where $\alpha>0, t>a, n \in N$, and $\alpha, a, t \in R$.

\section{Fractional Reduced Differential Transform Method (FRDTM)}

To illustrate the FRDTM, we give its definitions and properties. Consider a function of $(n+1)$ variables $u\left(t, x_{1}, x_{2}, \ldots, x_{n}\right)$, such that

$$
u\left(t, x_{1}, x_{2}, \ldots, x_{n}\right)=u_{1}\left(x_{1}\right) u_{2}\left(x_{2}\right) \cdots u_{n}\left(x_{n}\right) h(t),
$$

Then, from the properties of the one-dimensional differential transform method (DTM), we have

$$
\begin{aligned}
u & \left(t, x_{1}, x_{2}, \ldots, x_{n}\right) \\
& =\sum_{i_{1}=0}^{\infty} u_{1}\left(i_{1}\right) x_{1}^{i_{1}} \cdots \sum_{i_{n}=0}^{\infty} u_{n}\left(i_{n}\right) x_{n}^{i_{n}} \sum_{j=0}^{\infty} h(j) t^{j} \\
& =\sum_{i_{1}=0}^{\infty} \cdots \sum_{i_{n}=0}^{\infty} \sum_{j=0}^{\infty} U\left(i_{1}, \ldots, i_{n}, j\right) x_{1}^{i_{1}} \cdots x_{n}^{i_{n}} t^{j},
\end{aligned}
$$

where $U\left(i_{1}, i_{2}, \ldots, i_{n}, j\right)=u_{1}\left(i_{1}\right) u_{2}\left(i_{2}\right) \cdots u_{n}\left(i_{n}\right) h(j)$ is referred to as the spectrum of $u\left(t, x_{1}, x_{2}, \ldots, x_{n}\right)$. Also, the lowercase $u\left(t, x_{1}, x_{2}, \ldots, x_{n}\right)$ is used for the original function, while its fractional reduced transformed function is represented by the uppercase $U_{k}\left(x_{1}, x_{2}, \ldots, x_{n}\right)$, which is called the $T$-function.

3.1. FRDTM for Single-Term Time-Fractional Equations [17, 19, $23,24]$. Let $u\left(t, x_{1}, x_{2}, \ldots, x_{n}\right)$ be analytical and continuously differentiable with respect to $n+1$ variables $t, x_{1}, x_{2}, \ldots, x_{n}$, 
in the domain of interest; then FRDTM in $n$-dimensions of $u\left(t, x_{1}, x_{2}, \ldots, x_{n}\right)$ is given by

$$
\begin{aligned}
& U_{k}\left(x_{1}, x_{2}, \ldots, x_{n}\right) \\
& \quad=\frac{1}{\Gamma(k \alpha+1)}\left[\mathbf{D}_{t}^{\alpha k}\left(u\left(t, x_{1}, x_{2}, \ldots, x_{n}\right)\right)\right]_{t=t_{0}},
\end{aligned}
$$

where $k=0,1,2, \ldots$

The inverse FRDTM of $U_{k}\left(x_{1}, x_{2}, \ldots, x_{n}\right)$ is defined by

$$
u\left(t, x_{1}, x_{2}, \ldots, x_{n}\right):=\sum_{k=0}^{\infty} U_{k}\left(x_{1}, x_{2}, \ldots, x_{n}\right)\left(t-t_{0}\right)^{k \alpha} .
$$

From (5) and (6), we have

$$
\begin{aligned}
& u\left(t, x_{1}, x_{2}, \ldots, x_{n}\right)=\sum_{k=0}^{\infty} \frac{1}{\Gamma(k \alpha+1)} \\
& \cdot\left[\mathbf{D}_{t}^{\alpha k}\left(u\left(t, x_{1}, x_{2}, \ldots, x_{n}\right)\right)\right]_{t=t_{0}}\left(t-t_{0}\right)^{k \alpha} .
\end{aligned}
$$

In particular, for $t_{0}=0$, the above equation becomes

$$
\begin{aligned}
& u\left(t, x_{1}, x_{2}, \ldots, x_{n}\right) \\
& \quad=\sum_{k=0}^{\infty} \frac{1}{\Gamma(k \alpha+1)}\left[\mathbf{D}_{t}^{\alpha k}\left(u\left(t, x_{1}, x_{2}, \ldots, x_{n}\right)\right)\right]_{t=0} t^{k \alpha} .
\end{aligned}
$$

From the above definition, it can be seen that the concept of FRDTM is derived from the power series expansion of a function. Then the inverse transformation of the set of values $\left\{U_{k}\left(x_{1}, x_{2}, \ldots, x_{n}\right)\right\}_{k=0}^{m}$ gives approximate solution as

$$
\widetilde{u}_{m}\left(t, x_{1}, x_{2}, \ldots, x_{n}\right)=\sum_{k=0}^{m} U_{k}\left(x_{1}, x_{2}, \ldots, x_{n}\right) t^{k},
$$

where $m$ is the order of approximate solution. Therefore, the exact solution is given by

$$
u\left(t, x_{1}, x_{2}, \ldots, x_{n}\right)=\lim _{m \longrightarrow \infty} \tilde{u}_{m}\left(t, x_{1}, x_{2}, \ldots, x_{n}\right) .
$$

Table 1 gives some properties of FRDTM, where $\delta(k-r)$ is defined by

$$
\delta(k-r)= \begin{cases}1, & k=r, \\ 0, & k \neq r .\end{cases}
$$

where $w=w\left(t, x_{1}, x_{2}, \ldots, x_{n}\right), u=u\left(t, x_{1}, x_{2} \cdots, x_{n}\right), W_{k}=$ $W_{k}\left(x_{1}, x_{2}, \ldots, x_{n}\right)$, and $U_{k}=U_{k}\left(x_{1}, x_{2}, \ldots, x_{n}\right)$.

3.2. The New Modification. In this section, we modify the definition of FRDTM for single-term time-fractional differential equation to be effective for MT-TFDEs as follows:

$$
\begin{aligned}
& \sum_{i=1}^{n} \mathbf{D}_{t}^{\alpha_{i}} u(t, X)+a \frac{\partial^{\beta} u(t, X)}{\partial t^{\beta}} \\
& =b \sum_{j=1}^{m} \frac{\partial^{2} u(t, X)}{\partial x_{j}^{2}}+f(t, X),
\end{aligned}
$$

TABLE 1: Fundamental operations of FRDTM [23, 24, 29, 30].

\begin{tabular}{lc}
\hline Original function & Transformed function \\
\hline$w=c_{1} u \pm c_{2} v$ & $W_{k}=c_{1} U_{k} \pm c_{2} V_{k}$ \\
$w=u v$ & $W_{k}=\sum_{i=0}^{k} U_{i} V_{k-i}$ \\
$w=\mathbf{D}_{t}^{m \alpha} u$ & $W_{k}=\frac{\Gamma(k \alpha+m \alpha+1)}{\Gamma(k \alpha+1)} U(k+m)$ \\
$w=\frac{\partial^{h} u}{\partial x_{i}^{h}}$ & $W_{k}=\frac{\partial^{h} U_{k}}{\partial x_{i}^{h}}, \quad i=1,2, \ldots, n$ \\
$w=x_{i}^{m} t^{r}$ & $W_{k}=x_{i}^{m} \delta(k-r), \quad i=1,2, \ldots, n$ \\
$w=x_{i}^{m} t^{r} u$ & $W_{k}=x_{i}^{m} U_{k-r}, \quad i=1,2, \ldots, n$ \\
$w=\mathrm{e}^{\lambda t}$ & $W_{k}=\frac{\lambda^{k}}{k !}$ \\
\hline
\end{tabular}

where $X=\left(x_{1}, x_{2}, \ldots, x_{m}\right), n, m$, and $\beta \in N, t \in[0, T]$, $T \in R^{+}, x_{j} \in[0, L], j=\{1,2, \ldots, m\}$ and $L \in R^{+}, \alpha_{i}>0$, $i=\{1,2, \ldots, n\}$, with initial condition $u(0, X)=u_{0}(t, X)$ and arbitrary constants $a, b \in Z$ not both of them equal to zero.

Let $u(t, X)$ be analytical and continuously differentiable with respect to $m+1$ variables $t$ and $X$ in the domain of interest $D$; then the $\mathrm{m}$-FRDTM in $m$-dimensions of $u(t, X)$ is given by

$$
U_{k}(X)=\frac{1}{\Gamma\left(k \sum_{i=1}^{n} \alpha_{i}+1\right)}\left[\sum_{i=1}^{n} \mathbf{D}_{t}^{\alpha_{i} k}(u(t, X))\right]_{t=t_{0}},
$$

where $k=0,1,2, \ldots$

The inverse FRDTM of $U_{k}(X)$ is defined by

$$
u(t, X):=\sum_{k=0}^{\infty} U_{k}(X)\left(t-t_{0}\right)^{k \sum_{i=1}^{n} \alpha_{i}} .
$$

From (13) and (14), we obtain

$$
\begin{aligned}
u(t, X)= & \sum_{k=0}^{\infty} \frac{1}{\Gamma\left(k \sum_{i=1}^{n} \alpha_{i}+1\right)}\left[\sum_{i=1}^{n} \mathbf{D}_{t}^{\alpha_{i} k}(u(t, X))\right]_{t=t_{0}} \\
& \cdot\left(t-t_{0}\right)^{k \sum_{i=1}^{n} \alpha_{i}}
\end{aligned}
$$

In particular, for $t_{0}=0$, the above equation becomes

$$
\begin{aligned}
& u(t, X) \\
& =\sum_{k=0}^{\infty} \frac{1}{\Gamma\left(k \sum_{i=1}^{n} \alpha_{i}+1\right)}\left[\sum_{i=1}^{n} \mathbf{D}_{t}^{\alpha_{i} k}(u(t, X))\right]_{t=0} t^{k \sum_{i=1}^{n} \alpha_{i}} .
\end{aligned}
$$


Then the inverse transformation of the set of values $\left\{U_{k}(X)\right\}_{k=0}^{z}, z=0,1,2, \ldots$, gives the approximate solution of the function $u(t, X)$ as a finite power series, where $z$ is the order of approximate solution:

$$
\tilde{u}_{z}(t, X)=\sum_{k=0}^{z} U_{k}(X) t^{k \sum_{i=1}^{n} \alpha_{i}},
$$

where $U_{k}(X)$ in (17) for MT-TFDEs (12) can be defined as

$$
\begin{aligned}
& \sum_{i=1}^{n} \frac{\Gamma\left(k \alpha_{i}+1\right)}{\Gamma\left((k-1) \alpha_{i}+1\right)} U_{k}(X) \\
& \quad+a \frac{\Gamma(k \beta+1)}{\Gamma((k-1) \beta+1)} U_{k}(X) \\
& \quad b \sum_{j=1}^{m} \frac{\partial^{2} U_{(k-1)}(X)}{\partial x_{j}^{2}}+F(X),
\end{aligned}
$$

where $F(X)$ is the FRDTM of $f(x, t)$ and can be found using the operations from Table 1. This shows that the FRDTM is a special case of the power series expansion.

Equation (12) represents time-fractional diffusion-wave equation if $1<\alpha_{i}<2$ and a fractional diffusion equation if $0<\alpha_{i}<1$. When $\alpha_{i}=2$, Equation (12) represents a traditional wave equation, while if $\alpha_{i}=1$, it represents a traditional diffusion equation.

\section{Application and Numerical Results}

In this section, we consider three different applications to demonstrate the applicability of the m-FRDTM to MTTFDEs. Two applications in two-term (1+1)-dimensional time-fractional equations and the third one in three-term $(2+1)$-dimensional time-fractional equation.

4.1. Example 1. If $n=\beta=m=a=1$ in Equation (12), then the two-term (1+1)-dimensional time-fractional diffusion equation is [31]

$$
\mathbf{D}_{t}^{\alpha} u(x, t)+\frac{\partial u(x, t)}{\partial t}=\frac{\partial^{2} u(x, t)}{\partial x^{2}}+f(x, t)
$$

subject to initial condition

$$
u(x, 0)=u_{0}(x, t)=0,
$$

where $0 \leq t \leq 1,0 \leq x \leq 1,0<\alpha<1$, and

$$
f(x, t)=\left(2 t-t^{2}+\frac{2 t^{2-\alpha}}{\Gamma(3-\alpha)}\right) \mathrm{e}^{x} .
$$

Applying the appropriate properties given in Table 1 to Equation (19), we obtain the following recurrence relation:

$$
\begin{aligned}
& U_{k+1}(x) \\
& \quad=\frac{\Gamma(k \alpha+1)}{(k+1) \Gamma(k \alpha+1)+\Gamma((k+1) \alpha+1)}\left(\frac{\partial^{2} U_{k}(x)}{\partial x^{2}}+2 \mathrm{e}^{x} \delta(k\right. \\
& \left.-1)-\mathrm{e}^{x} \delta(k-2)+\frac{2 \mathrm{e}^{x} \delta(k-2+\alpha)}{\Gamma(3-\alpha)}\right),
\end{aligned}
$$

where $k=0,1,2, \ldots$

From (22) the inverse transform coefficients of $t^{k \alpha}$ are given below:

$$
\begin{aligned}
& U_{0}(x)=0, \\
& U_{1}(x)= \begin{cases}\frac{2 \mathrm{e}^{x}}{3}, & \alpha=2, \\
0, & \alpha \neq 2,\end{cases} \\
& U_{2}(x)= \begin{cases}\frac{4 \mathrm{e}^{x}}{21}, & \alpha=2, \\
\mathrm{e}^{x}, & \alpha \neq 2, \alpha \neq 1, \\
\frac{2 \mathrm{e}^{x} \Gamma(\alpha+1)}{2 \Gamma(\alpha+1)+\Gamma(2 \alpha+1)}, & \mathrm{e}^{x} \Gamma(2 \alpha+1)^{2}\end{cases} \\
& U_{3}(x)= \begin{cases}-\frac{\mathrm{e}^{x}}{(2 \Gamma(\alpha+1)+\Gamma(2 \alpha+1))(3 \Gamma(2 \alpha+1)+\Gamma(3 \alpha+1))} & \alpha \neq 0 \wedge \alpha \neq 1 \wedge \alpha \neq 2, \\
0, & \alpha=0,\end{cases}
\end{aligned}
$$

Continuing in the same manner and after a few iterations, the differential inverse transform of $\left\{U_{k}\right\}_{k=0}^{\infty}$ will give the following series solution: 


$$
\begin{aligned}
u(x, t) & =\sum_{k=0}^{\infty} U_{k}(x) t^{k \alpha}, \\
& =U_{0}(x)+U_{1}(x) t^{\alpha}+U_{2}(x) t^{2 \alpha}+U_{3}(x) t^{3 \alpha}+\cdots, \\
u_{3}(x, t) & = \begin{cases}\frac{5 \mathrm{e}^{x}}{6}, & \alpha=0, \\
t^{2} \mathrm{e}^{x}, & \alpha=1, \\
-\frac{1}{693} t^{2}\left(17 t^{4}-132 t^{2}-462\right) \mathrm{e}^{x}, & \alpha=2, \\
\frac{\mathrm{e}^{x} t^{2 \alpha}\left(2 \Gamma(\alpha+1)(3 \Gamma(2 \alpha+1)+\Gamma(3 \alpha+1))-\Gamma(2 \alpha+1)^{2} t^{\alpha}\right)}{(2 \Gamma(\alpha+1)+\Gamma(2 \alpha+1))(3 \Gamma(2 \alpha+1)+\Gamma(3 \alpha+1))}, & \alpha \neq 0, \alpha \neq 1, \alpha \neq 2 .\end{cases}
\end{aligned}
$$

If $\alpha=1$, then the FRDTM solution (24) gives the exact solution of nonfractional diffusion Equation (19) subject to (20):

$$
u(x, t)=t^{2} \mathrm{e}^{x}
$$

which is exactly the same result obtained in [31], while for the fractional order we can obtain the approximate solutions for different values of $\alpha$ :

$$
\begin{aligned}
& u_{3}(x, t)=\left(0.565779 t^{1.6}-0.0853873 t^{2.4}\right) e^{x}, \\
& \alpha=0.8, \\
& u_{3}(x, t)=\left(0.618599 t^{1.2}-0.084351 t^{1.8}\right) e^{x}, \\
& \alpha=0.6, \\
& u_{3}(x, t)=\left(0.655797 t^{0.8}-0.0822868 t^{1.2}\right) e^{x}, \\
& \alpha=0.4 .
\end{aligned}
$$

In general, the approximate solutions of (19) subject to (20) can be written as

$$
u_{3}(x, t)=\left(a t^{2 \alpha}-b t^{3 \alpha}\right) e^{x}, \quad 0<\alpha<1,
$$

where $a$ and $b \in R^{+}, 0.6 \leq a \leq 1$, and $0<b<0.2$.
The 3-dimensional plots of the FRDTM solutions of (19) with initial condition (20) are shown in Figure 1 for different values of $(\alpha=1,0.8,0.6,0.4)$. Figure 2 depicts solutions in 2 dimensional plots for different values of $(\alpha=1,0.8,0.6,0.4)$ for $t \in[0,1]$ and $x=0.5$. The solutions in 2-dimensional plots for $\alpha=0.8$ are depicted for different values of $x$ in Figure 3 .

4.2. Example 2. If $n=2, m=b=1$, and $a=0$ in Equation (12), then the two-term (1+1)-dimensional timefractional diffusion equation is [11]

$$
\mathbf{D}_{t}^{\alpha_{1}} u(x, t)+\mathbf{D}_{t}^{\alpha_{2}} u(x, t)=\frac{\partial^{2} u(x, t)}{\partial x^{2}}+f(x, t),
$$

subject to initial condition

$$
u(x, 0)=u_{0}(x, t)=x(1-x),
$$

where $0 \leq t \leq 0.5,0 \leq x \leq 1,0<\alpha_{i}<1, i=\{1,2\}$, and

$$
\begin{aligned}
f(x, t)= & \left(\frac{2 t^{2-\alpha_{1}}}{\Gamma\left(3-\alpha_{1}\right)}+\frac{2 t^{2-\alpha_{2}}}{\Gamma\left(3-\alpha_{2}\right)}\right)\left(x-x^{2}\right) \\
& +2\left(1+t^{2}\right) .
\end{aligned}
$$

Applying the appropriate properties given in Table 1 to Equation (28), we obtain the following recurrence relation:

$$
\begin{aligned}
U_{k+1}(x)= & \frac{\Gamma\left(k \alpha_{1}+1\right) \Gamma\left(k \alpha_{2}+1\right)}{\Gamma\left((k+1) \alpha_{1}+1\right) \Gamma\left(k \alpha_{2}+1\right)+\Gamma\left(k \alpha_{1}+1\right) \Gamma\left((k+1) \alpha_{1}+1\right)} \\
& \times\left[\left(x-x^{2}\right)\left(\frac{2 \delta\left(k-2+\alpha_{1}\right)}{\Gamma(3-\alpha)}+\frac{2 \delta\left(k-2+\alpha_{2}\right)}{\Gamma\left(3-\alpha_{2}\right)}\right)+2(\delta(k-2)+\delta(k))+\frac{\partial^{2} U_{k}(x)}{\partial x^{2}}\right],
\end{aligned}
$$




$$
\begin{aligned}
& U_{0}(x)=x(1-x), \\
& U_{1}(x)= \begin{cases}0, & \alpha_{1} \neq 2 \vee \alpha_{2} \neq 2 \\
\left(-\frac{2}{3}\right)(x-1) x, & \alpha_{1}=2 \vee \alpha_{2}=2,\end{cases} \\
& \begin{cases}\frac{2 \Gamma\left(\alpha_{2}+1\right)}{\Gamma\left(\alpha_{2}+1\right)+\Gamma\left(2 \alpha_{2}+1\right)}, & \alpha_{1}=0, \\
\frac{2 \Gamma\left(\alpha_{1}+1\right)}{\Gamma\left(\alpha_{1}+1\right)+\Gamma\left(2 \alpha_{1}+1\right)}, & \alpha_{2}=0\end{cases} \\
& \mid \begin{array}{l}
\Gamma\left(\alpha_{1}+1\right)+\Gamma\left(2 \alpha_{1}+1\right) \\
\frac{2\left(-x^{2}+x+1\right) \Gamma\left(\alpha_{2}+1\right)}{2 \Gamma\left(\alpha_{2}+1\right)+\Gamma\left(2 \alpha_{2}+1\right)},
\end{array} \quad \alpha_{1}=1 \\
& \begin{cases}\frac{2\left(-x^{2}+x+1\right) \Gamma\left(\alpha_{1}+1\right)}{2 \Gamma\left(\alpha_{1}+1\right)+\Gamma\left(2 \alpha_{1}+1\right)}, & \alpha_{2}=1,\end{cases} \\
& U_{2}(x)= \begin{cases}2 \Gamma\left(\alpha_{1}+1\right)+\Gamma\left(2 \alpha_{1}+1\right) & \alpha_{1}=\alpha_{2}=1, \\
-x^{2}+x+\frac{1}{2}, & \alpha_{2}=1,\end{cases} \\
& \left(-\frac{2}{3}\right)((x-1) x-1), \quad\left(\alpha_{1}=0 \wedge \alpha_{2}=1\right) \vee\left(\alpha_{1}=1 \wedge \alpha_{2}=0\right) \text {, } \\
& \begin{array}{ll}
\frac{2}{39}, & \left(\alpha_{1}=0 \wedge \alpha_{2}=2\right) \vee\left(\alpha_{2}=0 \wedge\right. \\
\left(\frac{1}{21}\right)(1-3(x-1) x), & \left(\alpha_{1}=2 \wedge \alpha_{2}=1\right) \vee\left(\alpha_{1}=1 \wedge\right. \\
\frac{2 \Gamma\left(\alpha_{1}+1\right) \Gamma\left(\alpha_{2}+1\right)}{\Gamma\left(2 \alpha_{1}+1\right) \Gamma\left(\alpha_{2}+1\right)+\Gamma\left(\alpha_{1}+1\right) \Gamma\left(2 \alpha_{2}+1\right)}, & \left(0<\alpha_{1}<1\right) \vee\left(0<\alpha_{2}<1\right),
\end{array} \\
& \int \frac{\left(-x^{2}+x+4\right) \Gamma\left(2 \alpha_{2}+1\right)}{\Gamma\left(2 \alpha_{2}+1\right)+\Gamma\left(3 \alpha_{2}+1\right)}, \quad \alpha_{1}=0 \\
& \frac{\left(-x^{2}+x+4\right) \Gamma\left(2 \alpha_{1}+1\right)}{\Gamma\left(2 \alpha_{1}+1\right)+\Gamma\left(3 \alpha_{1}+1\right)}, \quad \alpha_{2}=0 \\
& \begin{array}{ll}
-x^{2}+x+2, & \alpha_{1}=\alpha_{2}=0, \\
\frac{4 \Gamma\left(2 \alpha_{2}+1\right)\left(\Gamma\left(\alpha_{2}+1\right)+\Gamma\left(2 \alpha_{2}+1\right)\right)}{\left(2 \Gamma\left(\alpha_{2}+1\right)+\Gamma\left(2 \alpha_{2}+1\right)\right)\left(3 \Gamma\left(2 \alpha_{2}+1\right)+\Gamma\left(3 \alpha_{2}+1\right)\right)}, & \alpha_{1}=1,
\end{array} \\
& U_{3}(x)=\left\{\begin{array}{l}
\frac{4 \Gamma\left(2 \alpha_{1}+1\right)\left(\Gamma\left(\alpha_{1}+1\right)+\Gamma\left(2 \alpha_{1}+1\right)\right)}{\left(2 \Gamma\left(\alpha_{1}+1\right)+\Gamma\left(2 \alpha_{1}+1\right)\right)\left(3 \Gamma\left(2 \alpha_{1}+1\right)+\Gamma\left(3 \alpha_{1}+1\right)\right)}, \quad \alpha_{2}=1, \\
\frac{1}{3},
\end{array}\right. \\
& \begin{array}{ll}
\frac{1}{3}, & \alpha_{1}=\alpha_{2}=1, \\
\frac{26}{231}, & \left(\alpha_{1}=1 \wedge \alpha_{2}=2\right) \vee\left(\alpha_{1}=2 \wedge \alpha_{2}=1\right) \\
\left(\frac{1}{12}\right)(8-3(x-1) x), & \left(\alpha_{1}=0 \wedge \alpha_{2}=1\right) \vee\left(\alpha_{1}=1 \wedge \alpha_{2}=0\right) \\
\frac{4 \Gamma\left(2 \alpha_{1}+1\right) \Gamma\left(2 \alpha_{2}+1\right)}{\Gamma\left(3 \alpha_{1}+1\right) \Gamma\left(2 \alpha_{2}+1\right)+\Gamma\left(2 \alpha_{1}+1\right) \Gamma\left(3 \alpha_{2}+1\right)}, & \left(0<\alpha_{1}<1\right) \vee\left(0<\alpha_{2}<1\right)
\end{array}
\end{aligned}
$$

Continuing in the same manner and after a few iterations, the differential inverse transform of $\left\{U_{k}\right\}_{k=0}^{\infty}$ will give the following series solution:

$$
u(x, t)=\sum_{k=0}^{\infty} U_{k}(x) t^{k\left(\alpha_{1}+\alpha_{2}\right)},
$$

$$
\begin{aligned}
= & U_{0}(x)+U_{1}(x) t^{\left(\alpha_{1}+\alpha_{2}\right)}+U_{2}(x) t^{2\left(\alpha_{1}+\alpha_{2}\right)} \\
& +U_{3}(x) t^{3\left(\alpha_{1}+\alpha_{2}\right)}+\cdots .
\end{aligned}
$$




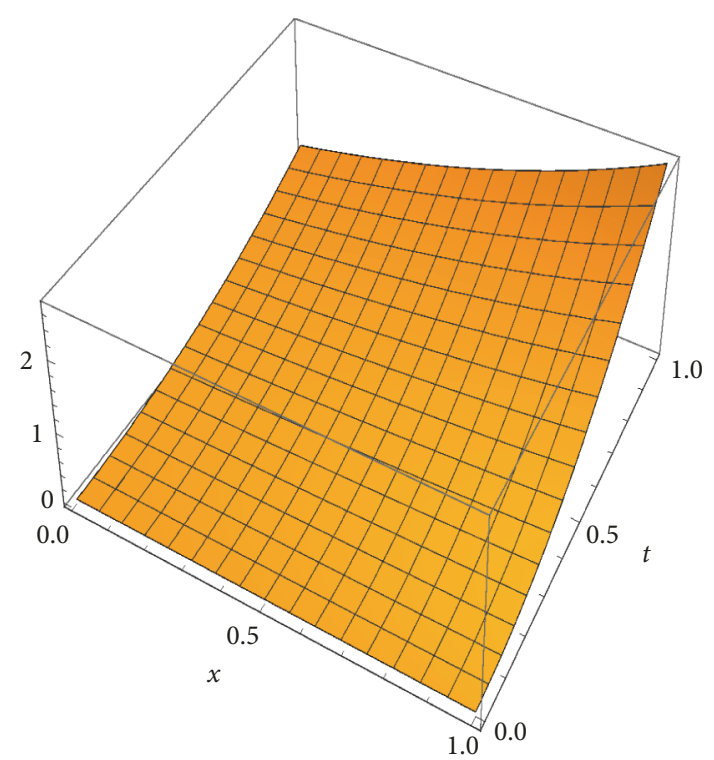

(a)

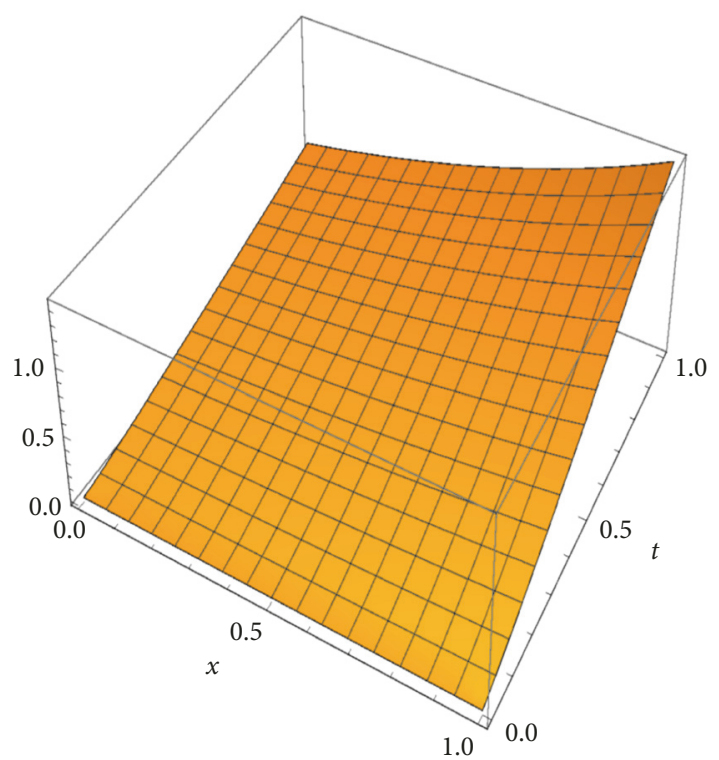

(c)

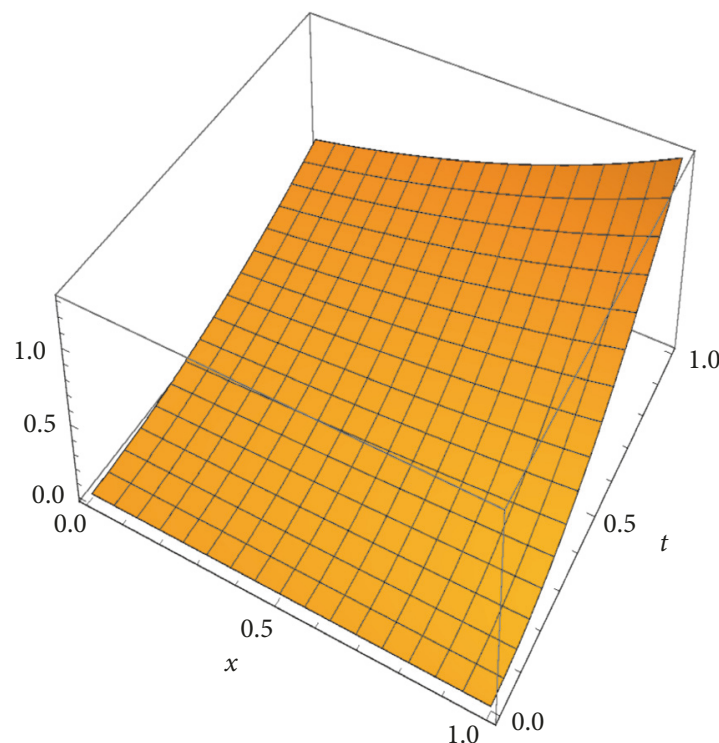

(b)

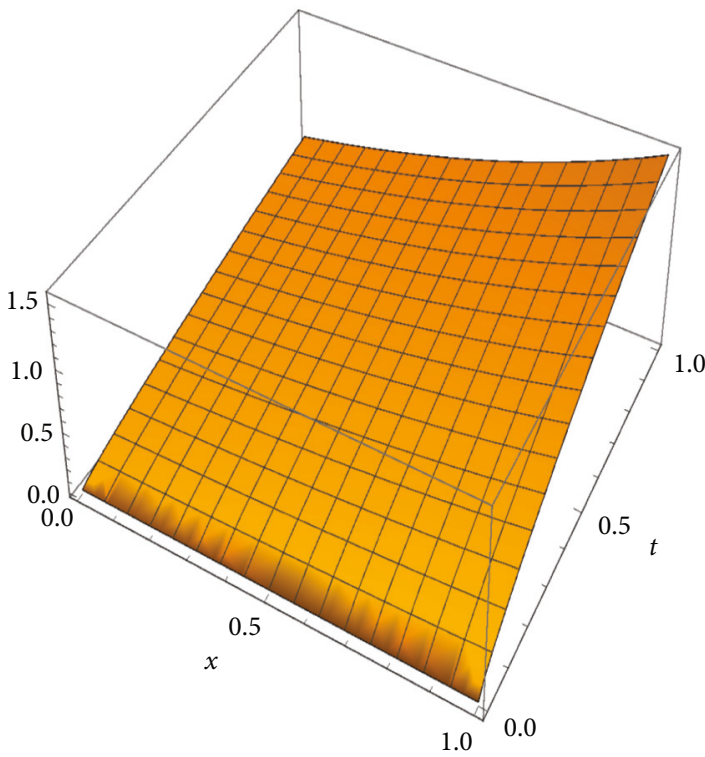

(d)

Figure 1: (a) $\alpha=1$ (exact), (b) $\alpha=0.8$ (3-term FRDTM), (c) $\alpha=0.6$, and (d) $\alpha=0.4$.

If $\alpha_{1}=\alpha_{2}=1$, then the third-approximate solution of the two-term time-nonfractional diffusion Equation (28) subject to $(29)$ is

$$
u_{3}(x, t)=\frac{t^{6}}{3}+\frac{1}{4} t^{4}\left(4\left(x-x^{2}\right)+2\right)+(1-x) x .
$$

The exact solution of nonfractional diffusion Equation (28) subject to (29) was given in [11] as

$$
u(x, t)=\left(t^{2}+1\right)\left(x-x^{2}\right) .
$$

The approximate solutions for different values of $\alpha_{1}$ and $\alpha_{2}$ are

$$
\begin{gathered}
u_{3}(x, t)=\frac{t^{6}}{3}+\frac{1}{4} t^{4}\left(4\left(x-x^{2}\right)+2\right)+(1-x) x, \\
\alpha_{1}=1, \alpha_{2}=1, \text { non-fractional, } \\
u_{3}(x, t)=t^{2.2}(x(0.67-0.67 x)+0.67)+x(1-x) \\
+0.667 t^{3.3}, \\
\alpha_{1}=1, \alpha_{2}=0.1, \text { fractional-one term, }
\end{gathered}
$$




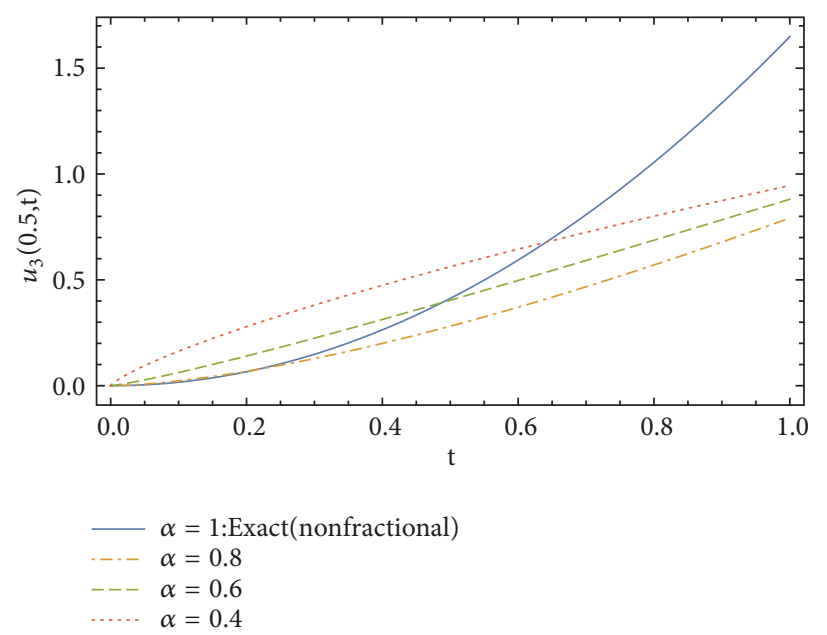

FIGURE 2: The 3-term FRDTM solutions $u_{3}$ for $\alpha=1$ (exact), $0.8,0.6,0.4 ; t \in[0,1]$; and $x=0.5$.

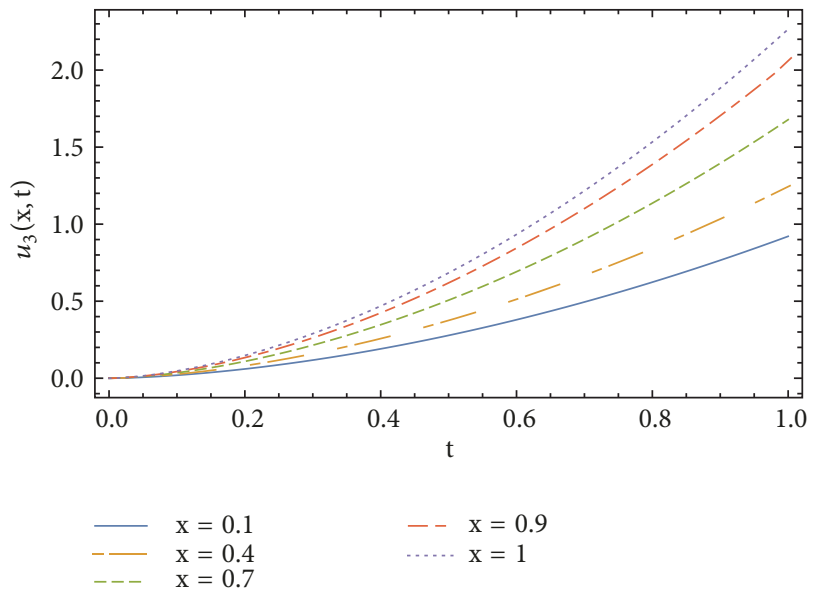

FIGURE 3: The 3-term FRDTM solutions $u_{3}$ for $x=0.1,0.4,0.7,0.9,1 ; \alpha=0.8 ; t \in[0,1]$.

$$
\begin{array}{r}
u_{3}(x, t)=1.53966 t^{2.7}+0.897383 t^{1.8}-(x-1) x, \\
\alpha_{1}=0.6, \alpha_{2}=0.3, \text { fractional-two term. }
\end{array}
$$

For simplicity if $\alpha_{1}=0.1(n), n=1,2, \ldots, 10$, then the closest approximate solutions of Equation (28) subject to (29) to the exact solution of nonfractional Equation (35) can be obtained when $\alpha_{2}=1$, then, when $\alpha_{2}=0.9$, then $\alpha_{2}=0.8, \ldots$, then $\alpha_{2}=0.1$. Similarly, if $\alpha_{2}=0.1(m), m=1,2, \ldots, 10$, then the closest approximate solutions of Equation (28) subject to (29) to the exact solution of nonfractional Equation (35) can be obtained when $\alpha_{1}=1$, then, when $\alpha_{1}=0.9$, then $\alpha_{1}=0.8, \ldots$, then $\alpha_{1}=0.1$.

We can note easily that the cases $\alpha_{1}=a$ and $\alpha_{2}=b$ will give the same approximate solutions when $\alpha_{1}=b$ and $\alpha_{2}=a$, for any constants $0<a \leq 1$ and $0<b \leq 1$, as we can see from Figure 5 which depicts solutions in 2-dimensional plots for different values of $\alpha_{1}$ and $\alpha_{2}$ for $t \in[0,0.5]$ and $x=0.1$.

The 3-dimensional plots of the FRDTM solutions of (28) with initial condition (29) are shown in Figure 4 for different values of $\alpha_{1}$ and $\alpha_{2}$.
4.3. Example 3. If $n=m=\beta=2, b=-1$, and $a=-1$ in Equation (12), then the three-term $(2+1)$-dimensional timefractional diffusion equation is [5]

$$
\begin{array}{r}
\mathbf{D}_{t}^{\alpha_{1}} u(x, y, t)+\mathbf{D}_{t}^{\alpha_{2}} u(x, y, t)-\frac{\partial^{\beta} u(x, y, t)}{\partial t^{\beta}} \\
=\frac{\partial^{2} u(x, y, t)}{\partial x^{2}}+\frac{\partial^{2} u(x, y, t)}{\partial y^{2}}+f(x, y, t),
\end{array}
$$

subject to initial condition

$$
u(x, y, 0)=u_{0}(x, y, t)=\sin (x) \sin (y)
$$

where $0<t \leq 1,0 \leq x, y \leq \pi, 0<\alpha_{1}<\alpha_{2}<1$, and

$$
\begin{aligned}
& f(x, y, t)=\sin (x) \sin (y)\left(2\left(1+t+t^{2}+t^{3}\right)\right. \\
& \left.\quad+\sum_{i=1}^{2}\left[\frac{t^{1-\alpha_{i}}}{\Gamma\left(2-\alpha_{i}\right)}+\frac{2 t^{2-\alpha_{i}}}{\Gamma\left(3-\alpha_{i}\right)}+\frac{6 t^{3-\alpha_{i}}}{\Gamma\left(4-\alpha_{i}\right)}\right]\right) .
\end{aligned}
$$




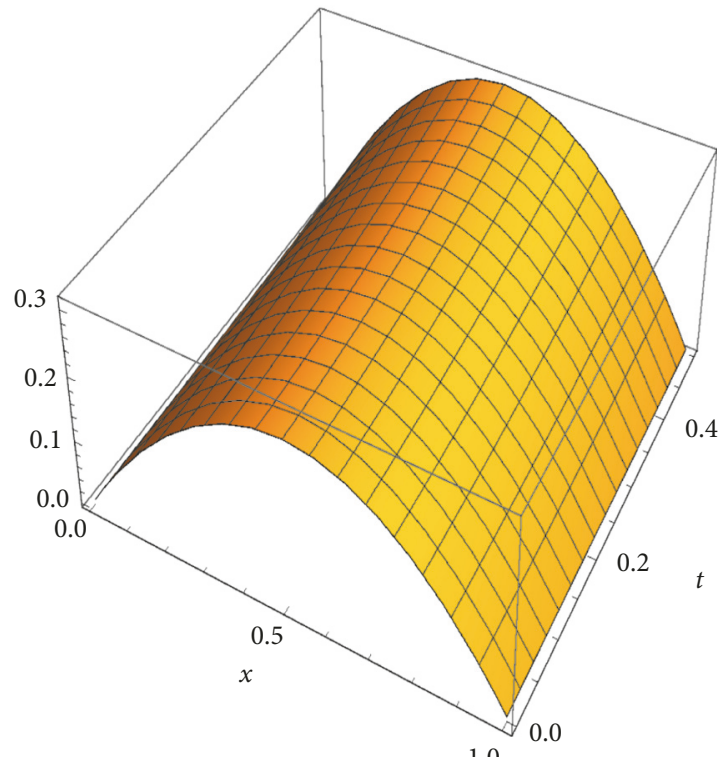

(a)

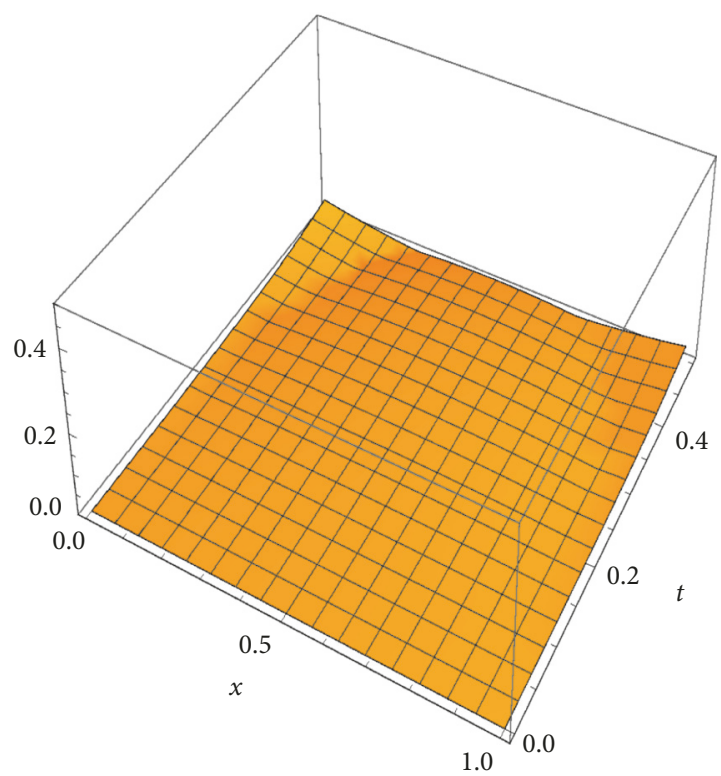

(c)

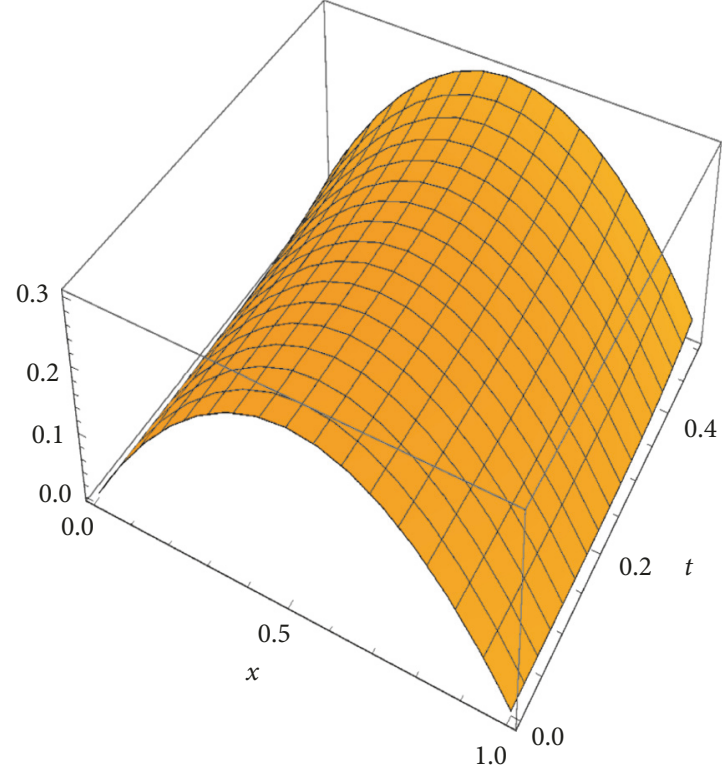

(b)

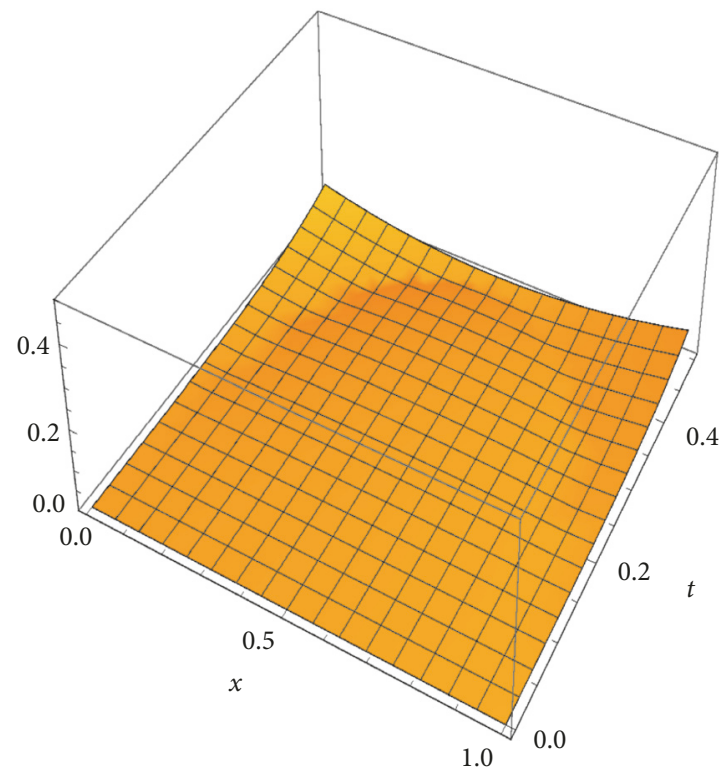

(d)

Figure 4: (a) Nonfractional (exact), (b) approximate solution $\alpha_{1}=\alpha_{2}=1$ (3-term FRDTM), (c) absolute error between nonfraction exact solution and nonfractional approximate solution when $\alpha_{1}=\alpha_{2}=1$, and (d) absolute error between nonfraction exact solution and fractional approximate solution when $\alpha_{1}=\alpha_{2}=0.9$.

Applying the appropriate properties given in Table 1 to Equation (37), we obtain the following recurrence relation:

$$
\begin{aligned}
& U_{k+1}(x, y)=\frac{1}{\Gamma\left(k \alpha_{2}+1\right) \Gamma\left(k \alpha_{1}+1\right) \Gamma(k \beta+1)} \\
& \quad \times\left(-\Gamma\left(k \alpha_{1}\right.\right. \\
& \quad+1) \Gamma\left(k \alpha_{2}+1\right) \Gamma((k+1) \beta+1)+\Gamma\left(k \alpha_{2}+1\right) \Gamma((k
\end{aligned}
$$

$\left.+1) \alpha_{1}+1\right) \Gamma(k \beta+1)+\Gamma\left(k \alpha_{1}+1\right) \Gamma\left((k+1) \alpha_{2}\right.$

$+1) \Gamma(k \beta+1))$

$\times[\sin (x)$

$\cdot \sin (y)\left(\sum_{i=1}^{2}\left(\frac{\delta\left(k-1+\alpha_{i}\right)}{\Gamma\left(2-\alpha_{i}\right)}+\frac{2 \delta\left(k-2+\alpha_{i}\right)}{\Gamma\left(3-\alpha_{i}\right)}\right.\right.$ 

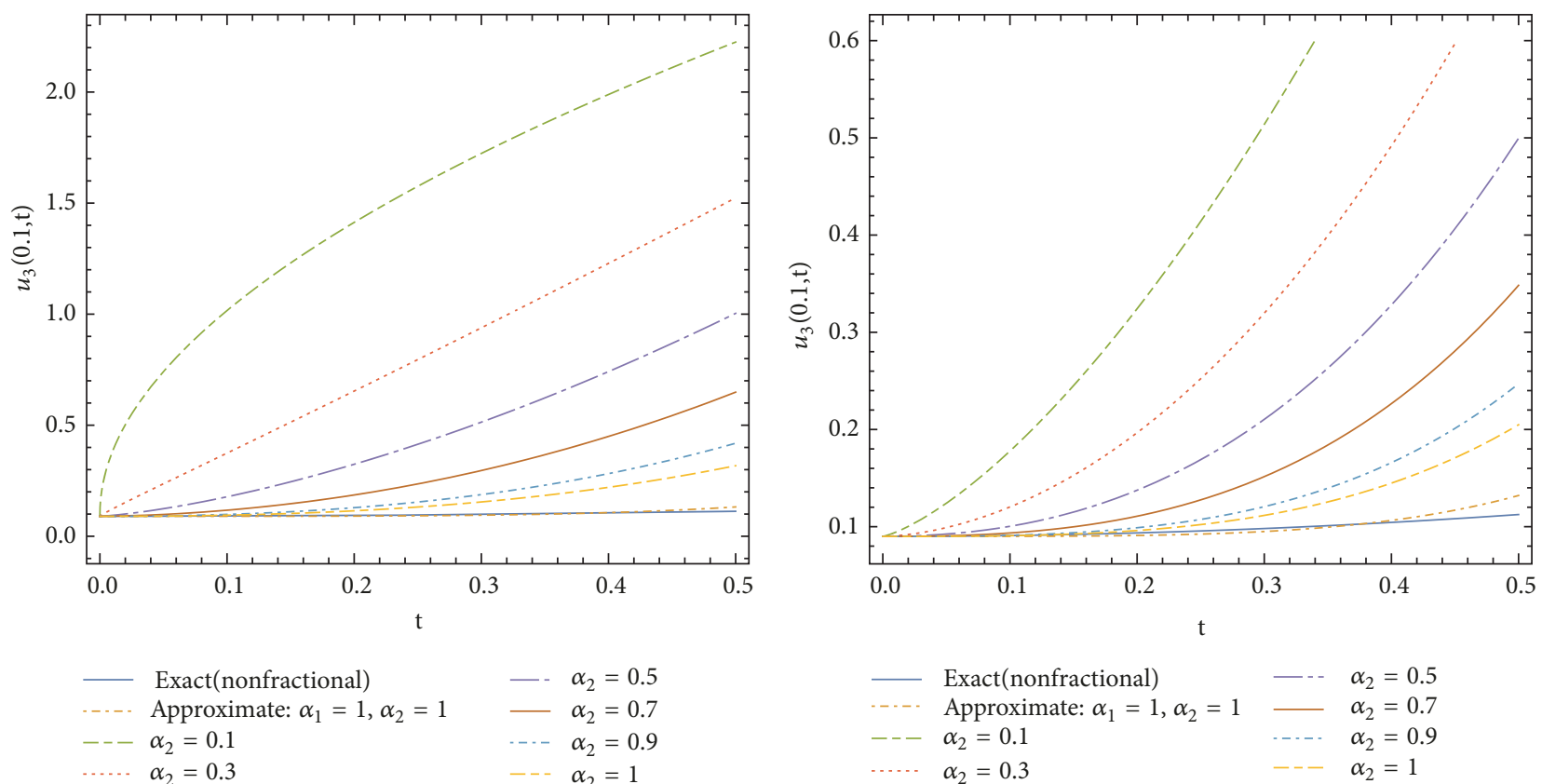

(a)

(b)

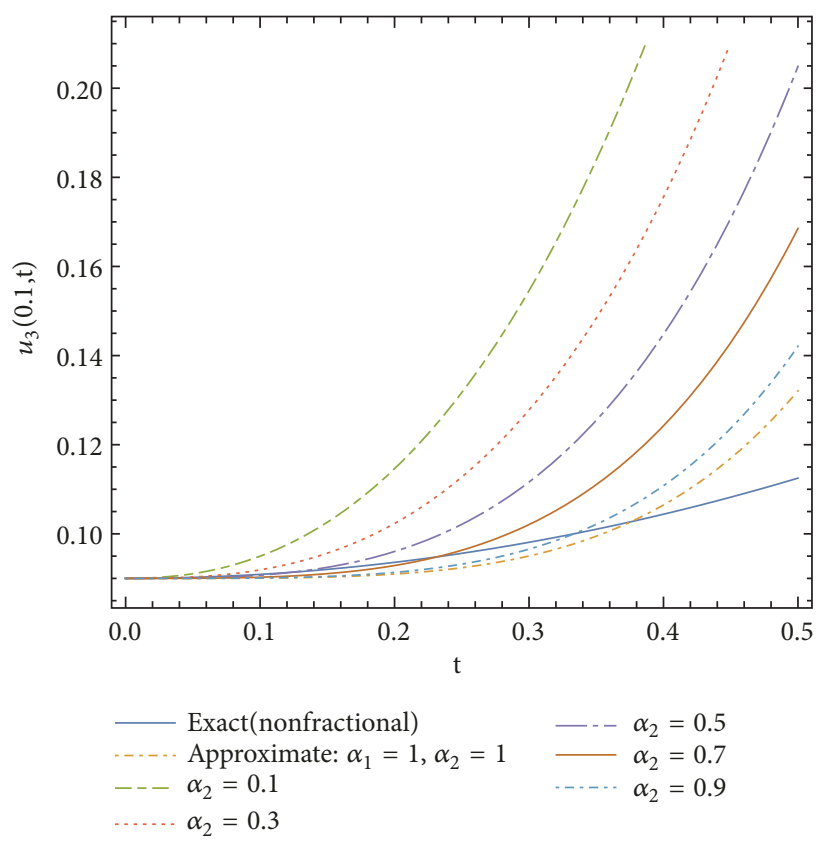

(c)

FIGURE 5: The 3-term FRDTM solutions $u_{3}$. (a) constant $\alpha_{1}=0.1$ and different values of $\alpha_{2}$, (b) constant $\alpha_{1}=0.5$ and different values of $\alpha_{2}$, and (c) constant $\alpha_{1}=1$ and different values of $\alpha_{2} ; t \in[0,0.5]$; and $x=0.1$.

$$
\begin{aligned}
& \left.+\frac{6 \delta\left(k-3+\alpha_{i}\right)}{\Gamma\left(4-\alpha_{i}\right)}\right)+2(\delta(k)+\delta(k-1)+\delta(k \\
& \left.-2)+\delta(k-3)))+\frac{\partial^{2} U_{k}(x, y)}{\partial^{x}}+\frac{\partial^{2} U_{k}(x, y)}{\partial^{y}}\right],
\end{aligned}
$$

where $k=0,1,2, \ldots$
The differential inverse transform of $\left\{U_{k}\right\}_{k=0}^{\infty}$ will give the following series solution:

$$
\begin{aligned}
u(x, y, t)= & \sum_{k=0}^{\infty} U_{k}(x, y) t^{k\left(\alpha_{1}+\alpha_{2}\right)} \\
= & U_{0}(x)+U_{1}(x) t^{\left(\alpha_{1}+\alpha_{2}\right)}+U_{2}(x) t^{2\left(\alpha_{1}+\alpha_{2}\right)} \\
& +U_{3}(x) t^{3\left(\alpha_{1}+\alpha_{2}\right)}+\cdots
\end{aligned}
$$




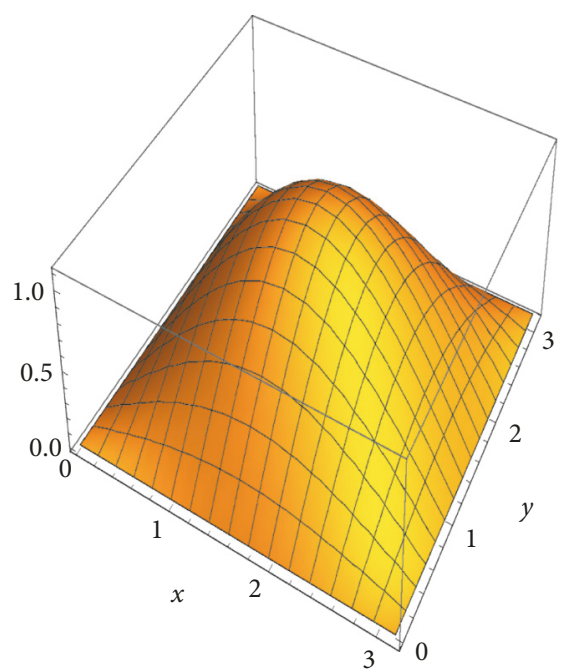

(a)

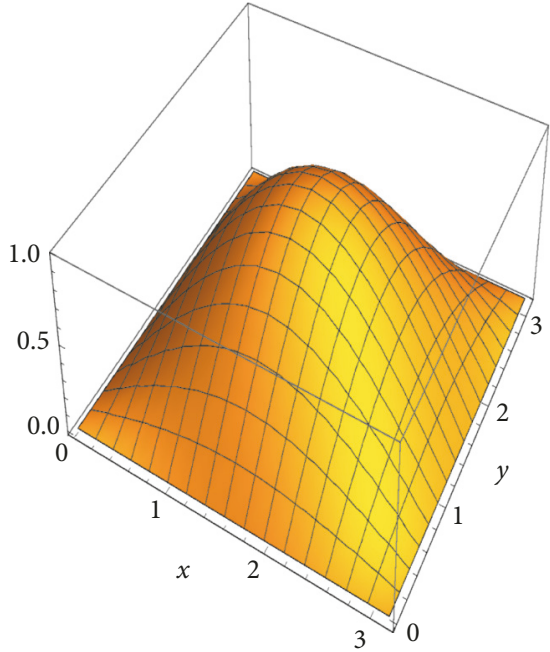

(b)

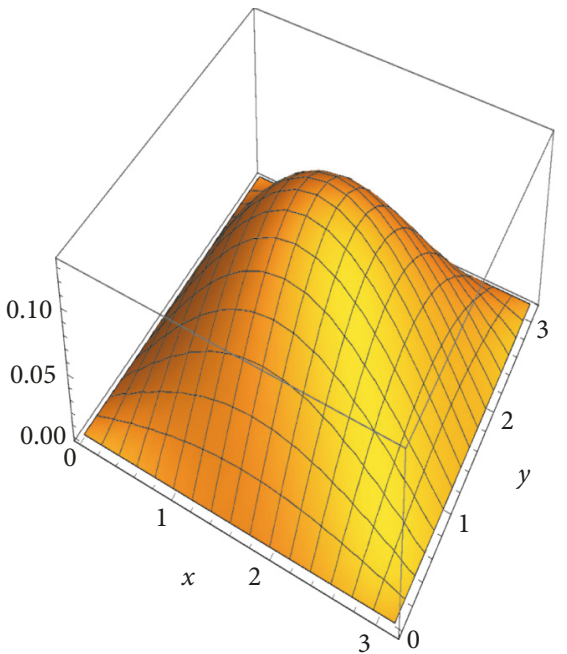

(c)

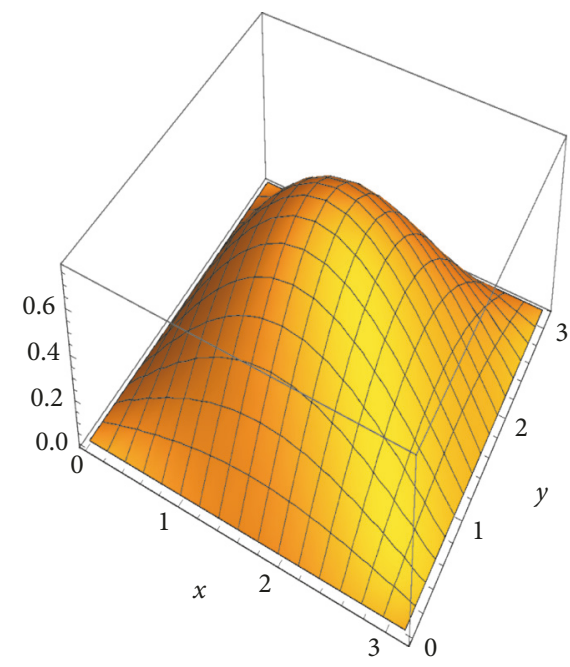

(d)

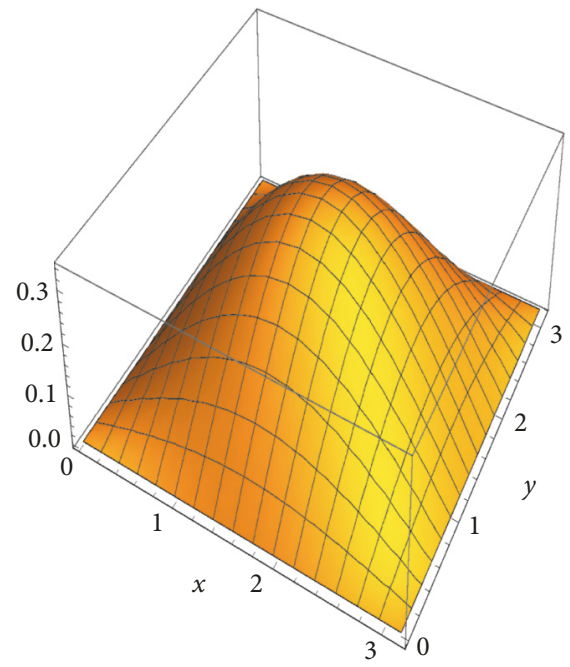

(e)

Figure 6: (a) Nonfractional (exact), (b) $\alpha_{1}=0.1, \alpha_{2}=0.4$ (3-term FRDTM), (c) absolute error between exact (nonfractional) solution and 3-term FRDTM approximate solution $\alpha_{1}=0.1, \alpha_{2}=0.4$, (d) $\alpha_{1}=0.8, \alpha_{2}=1$ (3-term FRDTM), and (e) absolute error between exact (nonfractional) solution and 3-term FRDTM approximate solution $\alpha_{1}=0.8, \alpha_{2}=1$.

The approximate solutions for different values of $\alpha_{1}$ and $\alpha_{2}$, $\beta=2$, are

$$
\begin{aligned}
& u_{3}(x, y, t)=\left\{\left(-0.515023 t^{5.4}-3.91582 t^{3.6}\right.\right. \\
& \left.\left.-14.5738 t^{1.8}+1 .\right) \sin (x) \sin (y)\right\}, \\
& \alpha_{1}=0.8, \alpha_{2}=1, \\
& u_{3}(x, y, t)=\left\{\left(-0.0855716 t^{0.33}-0.199195 t^{0.22}+1 .\right)\right. \\
& \cdot \sin (x) \sin (y)\}, \quad \alpha_{1}=0.01, \alpha_{2}=0.1, \\
& u_{3}(x, y, t)=\left\{\left(-0.0858234 t^{1.2}-0.199217 t^{0.8}+1 .\right)\right. \\
& \cdot \sin (x) \sin (y)\} \quad \alpha_{1}=0.1, \alpha_{2}=0.3 .
\end{aligned}
$$

The exact solution of nonfractional time-diffusion Equation (37) subject to (38) was given in [5] as

$$
u(x, y, t)=\left(1+t+t^{2}+t^{3}\right) \sin (x) \sin (y) .
$$

The 3-dimensional plots of the FRDTM solutions of (37) with initial condition (38) are shown in Figure 6 for different values of $\alpha_{1}$ and $\alpha_{2}, \beta=2,0 \leq x, y \leq \pi$, and $t=0.1$. Figure 7 depicts solutions in 2-dimensional plots for different values of $\alpha_{1}$ and $\alpha_{2}$ for $x \in[0, \pi], t=0.1$, and $\beta=2$.

\section{Conclusions}

In this study, we introduced the m-FRDTM for the first time to handle MT-TFDEs. In Example 1 we obtained the exact solution of the nonfractional equation using the $\mathrm{m}$ FRDTM and compared it with the approximate solutions of 


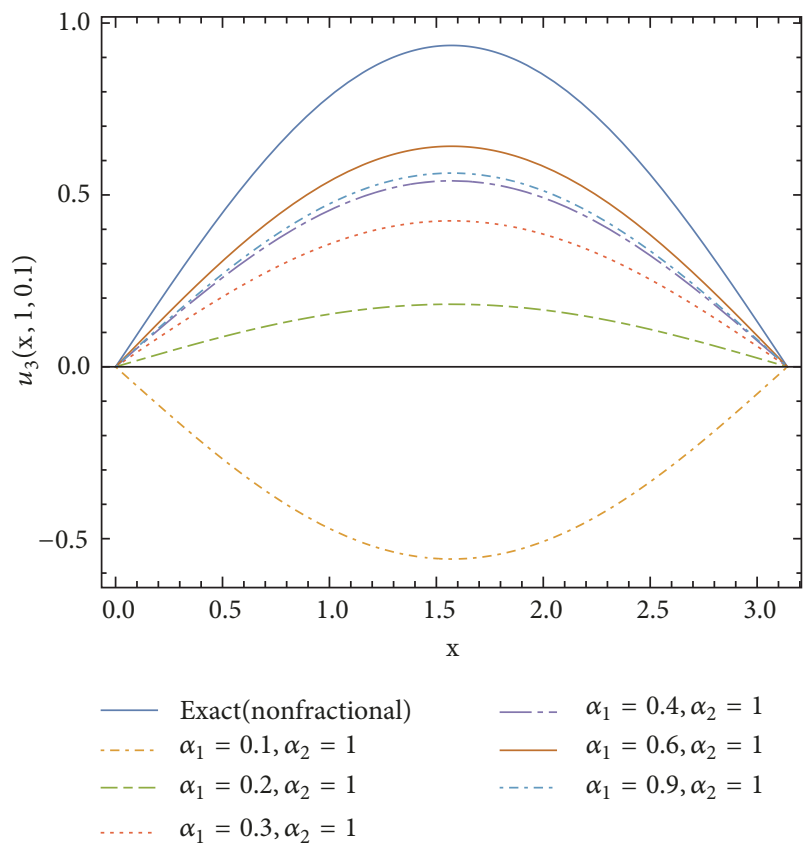

(a)

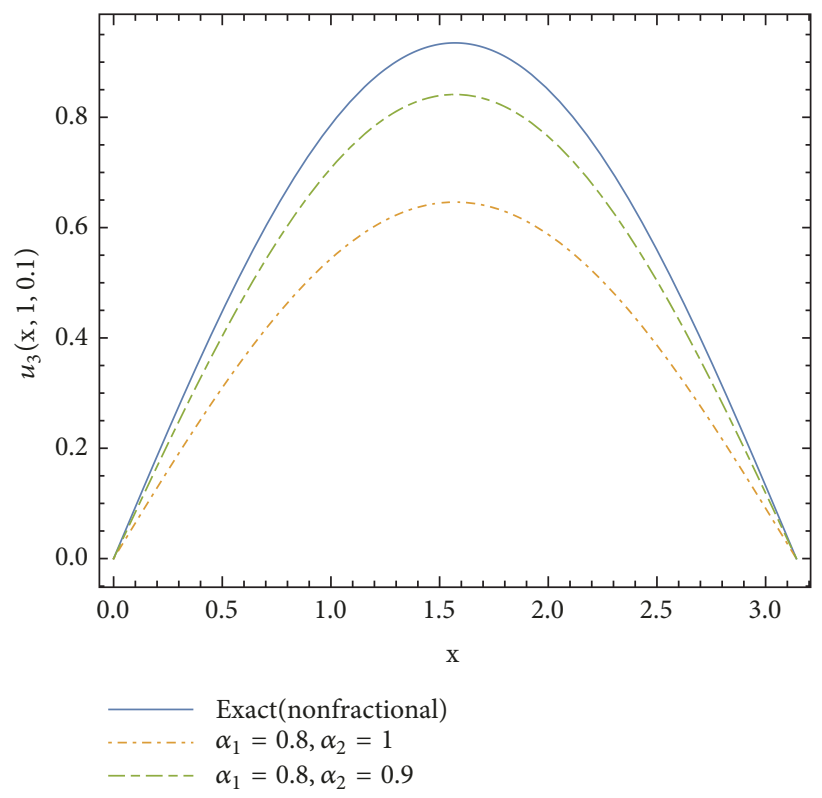

(b)

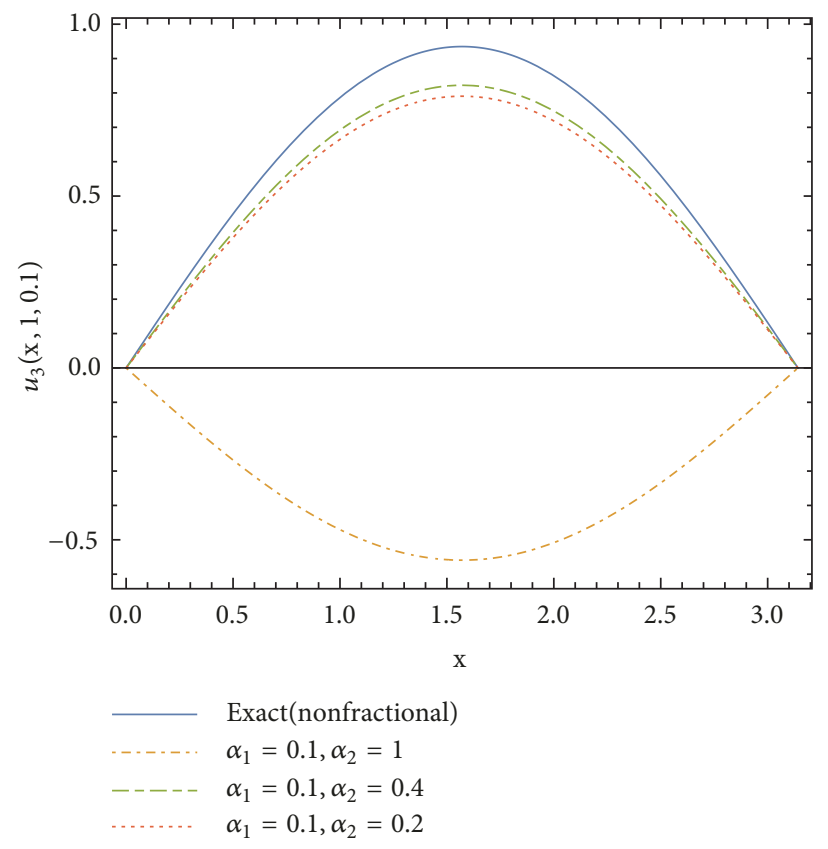

(c)

Figure 7: The 3-term FRDTM solutions $u_{3}$. (a) constant $\alpha_{2}=1$ and different values of $\alpha_{1}$, (b) constant $\alpha_{1}=0.8$ and different values of $\alpha_{2}$, and (c) constant $\alpha_{1}=0.1$ and different values of $\alpha_{2} ; x \in[0, \pi], y=1$; and $t=0.1$.

fractional equation for different orders, while in Examples 2 and 3 we obtained the approximate solutions of fractional equations using the m-FRDTM and compared them with the exact solutions of the nonfractional equations for different orders. Figures 1, 4, and 6 in three dimensions and Figures 2, 5, and 7 in two dimensions demonstrated that the m-FRDTM is very promising. Our future work will include the different types of multiterm fractional equations.

\section{Data Availability}

The datasets used and analysed during the current study are available from the corresponding author upon reasonable request.

\section{Conflicts of Interest}

The authors declare that they have no competing interests. 


\section{Authors' Contributions}

All authors read and approved the final manuscript.

\section{Acknowledgments}

This work is funded by the Deanship of Scientific Research, King Faisal University, through the Nasher track (Grant no. 186087). Salah Abuasad is thankful to the Deanship of Scientific Research, King Faisal University. The authors would like to thank the respected reviewers for their valuable comments and suggestions.

\section{References}

[1] S. Abuasad and I. Hashim, "Homotopy decomposition method for solving one-dimensional time-fractional diffusion equation," in Proceedings of the AIP Conference, vol. 1940, p. 020126, AIP Publishing, Selangor, Malaysia, 2018.

[2] S. Abuasad and I. Hashim, "Homotopy decomposition method for solving higher-order time- fractional diffusion equation via modified beta derivative," Sains Malaysiana, vol. 47, no. 11, pp. 2899-2905, 2018.

[3] H. Jiang, F. Liu, I. Turner, and K. Burrage, "Analytical solutions for the multi-term time-fractional diffusion-wave/diffusion equations in a finite domain," Computers \& Mathematics with Applications, vol. 64, no. 10, pp. 3377-3388, 2012.

[4] S. Abuasad, A. Yildirim, I. Hashim, S. Abdul Karim, and J. Gómez-Aguilar, "Fractional multi-step differential transformed method for approximating a fractional stochastic sis epidemic model with imperfect vaccination," International Journal of Environmental Research and Public Health, vol. 16, no. 6, p. 973, 2019.

[5] L. Zhao, F. Liu, and V. V. Anh, "Numerical methods for the twodimensional multi-term time-fractional diffusion equations," Computers \& Mathematics with Applications. An International Journal, vol. 74, no. 10, pp. 2253-2268, 2017.

[6] J. T. Edwards, N. J. Ford, and A. C. Simpson, "The numerical solution of linear multi-term fractional differential equations: systems of equations," Journal of Computational and Applied Mathematics, vol. 148, no. 2, pp. 401-418, 2002.

[7] V. Daftardar-Gejji and S. Bhalekar, "Solving multi-term linear and non-linear diffusion-wave equations of fractional order by adomian decomposition method," Applied Mathematics and Computation, vol. 202, no. 1, pp. 113-120, 2008.

[8] V. Daftardar-Gejji and S. Bhalekar, "Boundary value problems for multi-term fractional differential equations," Journal of Mathematical Analysis and Applications, vol. 345, no. 2, pp. 754$765,2008$.

[9] V. Srivastava and K. N. Rai, "A multi-term fractional diffusion equation for oxygen delivery through a capillary to tissues," Mathematical and Computer Modelling, vol. 51, no. 5-6, pp. 616624, 2010.

[10] A. M. El-Sayed, I. L. El-Kalla, and E. A. Ziada, "Analytical and numerical solutions of multi-term nonlinear fractional orders differential equations," Applied Numerical Mathematics, vol. 60, no. 8, pp. 788-797, 2010.

[11] B. Jin, R. Lazarov, Y. Liu, and Z. Zhou, "The Galerkin finite element method for a multi-term time-fractional diffusion equation," Journal of Computational Physics, vol. 281, pp. 825843, 2015.
[12] M. Dehghan, M. Safarpoor, and M. Abbaszadeh, "Two highorder numerical algorithms for solving the multi-term time fractional diffusion-wave equations," Journal of Computational and Applied Mathematics, vol. 290, pp. 174-195, 2015.

[13] M. Zheng, F. Liu, V. Anh, and I. Turner, "A high-order spectral method for the multi-term time-fractional diffusion equations," Applied Mathematical Modelling: Simulation and Computation for Engineering and Environmental Systems, vol. 40, no. 7-8, pp. 4970-4985, 2016.

[14] H. Chen, S. Lü, and W. Chen, "A unified numerical scheme for the multi-term time fractional diffusion and diffusion-wave equations with variable coefficients," Journal of Computational and Applied Mathematics, vol. 330, pp. 380-397, 2018.

[15] Y. Keskin and G. Oturanç, “The reduced differential transform method: a new approach to fractional partial differential equations," Nonlinear Sciences Letters A, vol. 1, no. 2, pp. 207-217, 2010.

[16] P. K. Gupta, "Approximate analytical solutions of fractional Benney-Lin equation by reduced differential transform method and the homotopy perturbation method," Computers \& Mathematics with Applications, vol. 61, no. 9, pp. 2829-2842, 2011.

[17] V. K. Srivastava, S. Kumar, M. K. Awasthi, and B. K. Singh, "Two-dimensional time fractional-order biological population model and its analytical solution," Egyptian Journal of Basic and Applied Sciences, vol. 1, no. 1, pp. 71-76, 2014.

[18] M. S. Rawashdeh, "A new approach to solve the fractional Harry Dym equation using the FRDTM," International Journal of Pure and Applied Mathematics, vol. 95, no. 4, pp. 553-556, 2014.

[19] B. K. Singh and V. K. Srivastava, "Approximate series solution of multi-dimensional, time fractional-order (heat-like) diffusion equations using FRDTM," Royal Society Open Science, vol. 2, no. 4, Article ID 140511, 2015.

[20] A. Saravanan and N. Magesh, "An efficient computational technique for solving the Fokker-Planck equation with space and time fractional derivatives," Journal of King Saud University - Science, vol. 28, no. 2, pp. 160-166, 2016.

[21] B. K. Singh, "Fractional reduced differential transform method for numerical computation of a system of linear and nonlinear fractional partial differential equations," International Journal of Open Problems in Computer Science and Mathematics, vol. 9, no. 3, pp. 20-38, 2016.

[22] M. S. Rawashdeh, "A reliable method for the space-time fractional Burgers and time-fractional Cahn-Allen equations via the FRDTM," Advances in Difference Equations, Paper No. 99, 14 pages, 2017.

[23] M. Arshad, D. Lu, and J. Wang, “ $(N+1)$-dimensional fractional reduced differential transform method for fractional order partial differential equations," Communications in Nonlinear Science and Numerical Simulation, vol. 48, pp. 509-519, 2017.

[24] S. Abuasad, K. Moaddy, and I. Hashim, "Analytical treatment of two-dimensional fractional Helmholtz equations," Journal of King Saud University - Science, 2018.

[25] I. Podlubny, Fractional Differential Equations, vol. 198 of Mathematics in Science and Engineering, Academic Press, San Diego, Calif, USA, 1999.

[26] K. S. Miller and B. Ross, An Introduction to the Fractional Calculus and Fractional Differential Equations, A Wiley-Interscience Publication, John Wiley \& Sons, New York, NY, USA, 1993.

[27] K. B. Oldham and J. Spanier, The Fractional Calculus Theory And Applications of Differentiation And Integration to Arbitrary Order, vol. 111, Academic Press, New York, NY, USA, 1974. 
[28] M. Caputo, "Linear models of dissipation whose $\mathrm{q}$ is almost frequency independent-ii," Geophysical Journal International, vol. 13, no. 5, pp. 529-539, 1967.

[29] Y. Keskin and G. Oturanç, "Reduced differential transform method for partial differential equations," International Journal of Nonlinear Sciences and Numerical Simulation, vol. 10, no. 6, pp. 741-749, 2009.

[30] I. A.-H. Hassan, "Application to differential transformation method for solving systems of differential equations," Applied Mathematical Modelling, vol. 32, no. 12, pp. 2552-2559, 2008.

[31] F. Liu, M. M. Meerschaert, R. J. McGough, P. Zhuang, and Q. Liu, "Numerical methods for solving the multi-term timefractional wave-diffusion equation," Fractional Calculus and Applied Analysis, vol. 16, no. 1, pp. 9-25, 2013. 


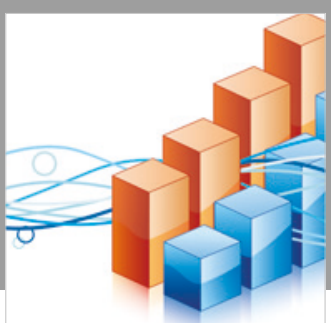

Advances in

Operations Research

\section{-n-m}
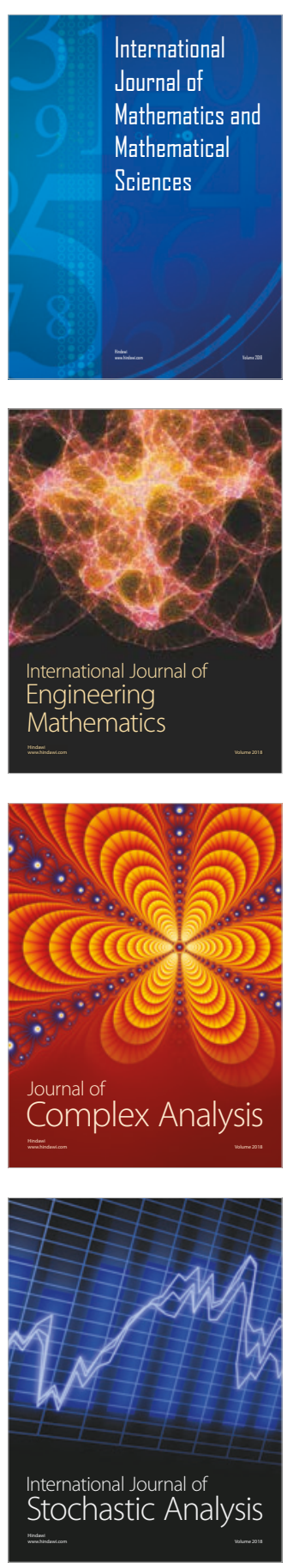
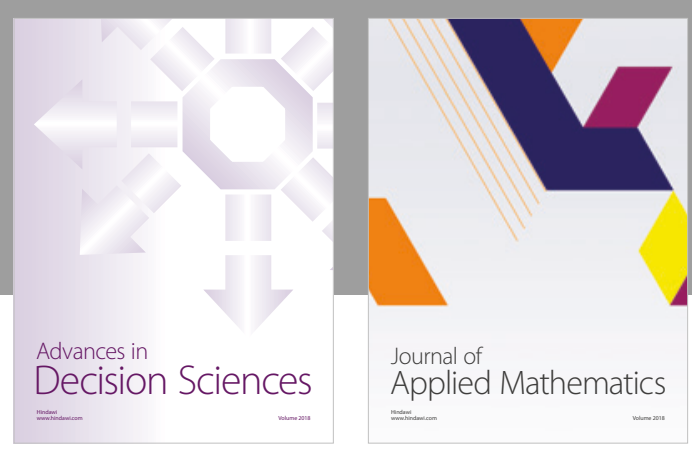

Journal of

Applied Mathematics
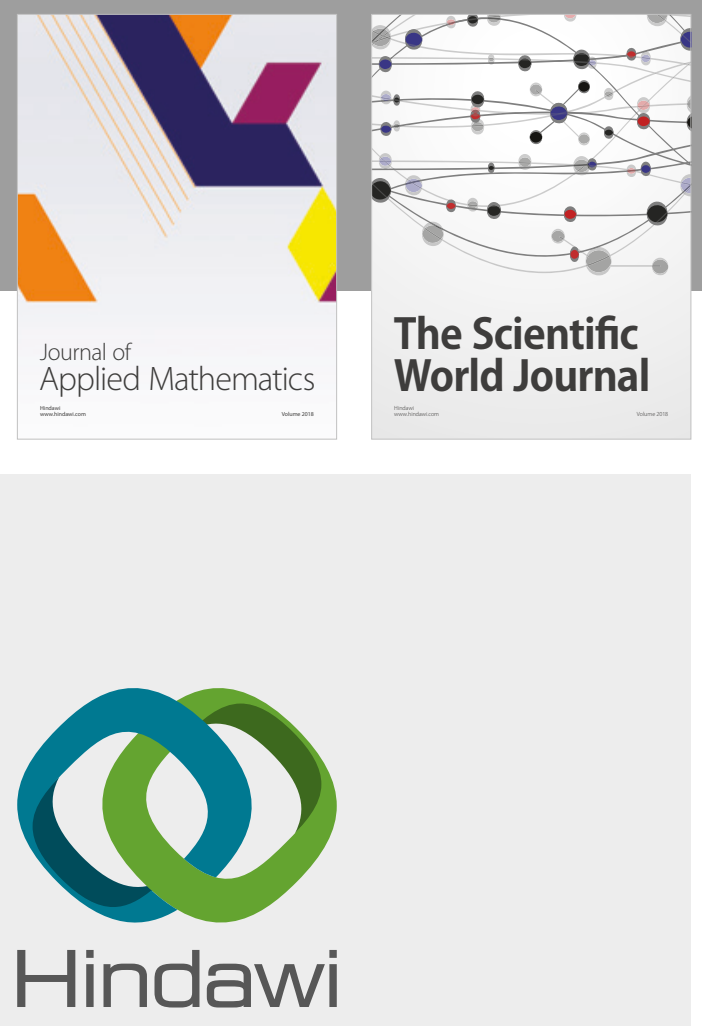

Submit your manuscripts at

www.hindawi.com

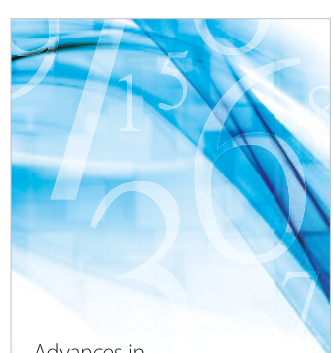

Advances in
Numerical Analysis
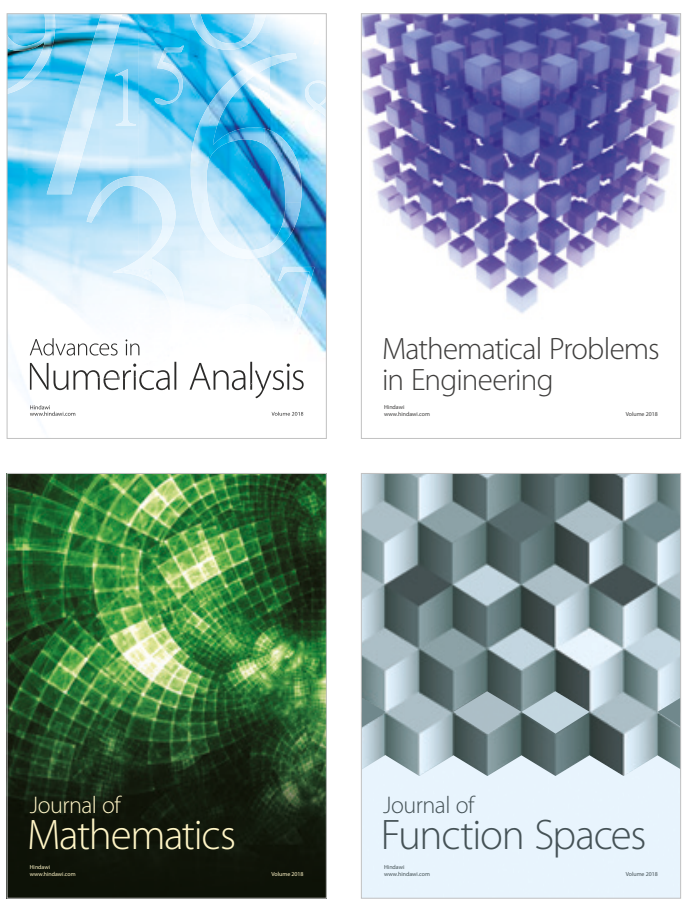

Mathematical Problems in Engineering

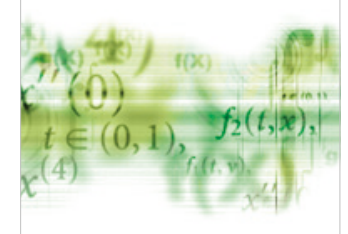

International Journal of

Differential Equations

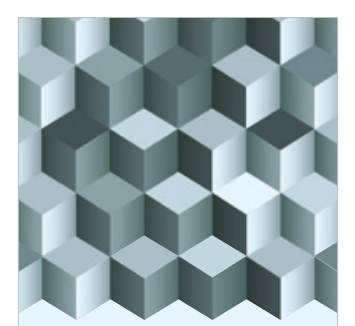

Journal of

Function Spaces

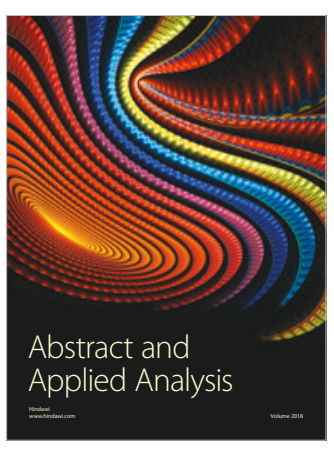

The Scientific

World Journal

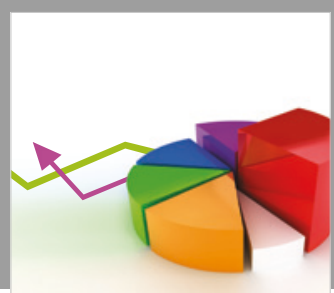

Journal of

Probability and Statistics
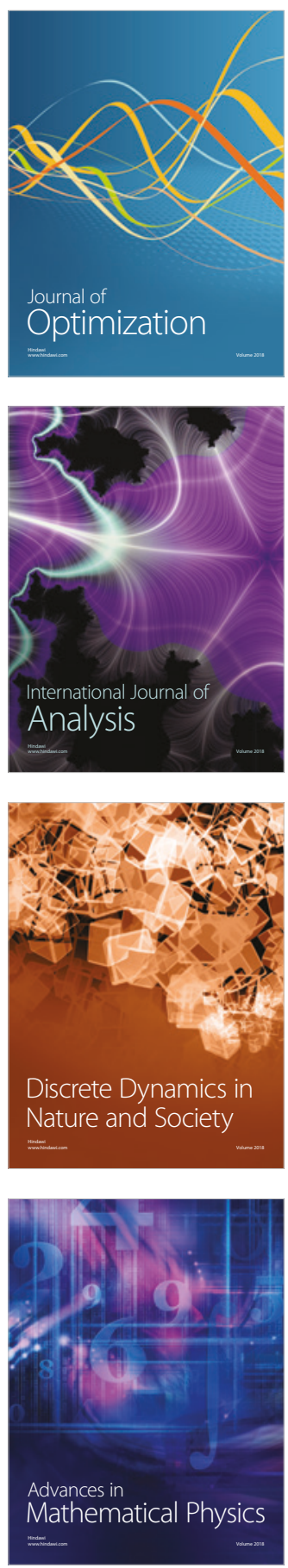